PROCEEDINGS OF THE

AMERICAN MATHEMATICAL SOCIETY

Volume 131, Number 2, Pages 577-585

S 0002-9939(02)06579-6

Article electronically published on June 18, 2002

\title{
MULTIPLE SYMMETRIC POSITIVE SOLUTIONS OF A CLASS OF BOUNDARY VALUE PROBLEMS FOR HIGHER ORDER ORDINARY DIFFERENTIAL EQUATIONS
}

\author{
JOHN R. GRAEF, CHUANXI QIAN, AND BO YANG \\ (Communicated by Carmen C. Chicone)
}

\begin{abstract}
In this paper, the authors consider the boundary value problem$$
x^{(2 m)}(t)+(-1)^{m+1} f(x(t))=0, \quad 0<t<1,
$$

$$
x^{(2 i)}(0)=x^{(2 i)}(1)=0, \quad i=0,1,2, \cdots, m-1,
$$

and give sufficient conditions for the existence of any number of symmetric positive solutions of $(\mathrm{E})-(\mathrm{B})$. The relationships between the results in this paper and some recent work by Henderson and Thompson (Proc. Amer. Math. Soc. 128 (2000), 2373-2379) are discussed.
\end{abstract}

\section{INTRODUCTION}

Consider the boundary value problem consisting of the even order nonlinear ordinary differential equation

$$
x^{(2 m)}(t)+(-1)^{m+1} f(x(t))=0, \quad 0<t<1,
$$

and the boundary conditions

$$
x^{(2 i)}(0)=x^{(2 i)}(1)=0, \quad i=0,1,2, \cdots, m-1,
$$

where $f: R \rightarrow[0,+\infty)$ is continuous. A special case of problem (1)-(2) is

$$
\begin{gathered}
x^{\prime \prime}(t)+f(x(t))=0, \quad 0<t<1, \\
x(0)=x(1)=0 .
\end{gathered}
$$

Boundary value problems for linear and nonlinear differential equations are important because of their many applications to physical, biological, and chemical phenomena. They are interesting as well from a theoretical perspective. There has been a great deal of research work on the existence of positive solutions for BVPs, and we cite as recent examples the papers of Agarwal and Wong 3], Avery, Davis, and Henderson 4, Baxley and Haywood [5, 6], Eloe et al. 7, 8], Erbe, Hu, and Wang [9], Henderson and Thompson [10, Ma, Zhang, and Fu 12], and Wong and Agarwal [13. Additional results and extensive bibliographies can be found in the recent monographs by Agarwal 1] and Agarwal, O'Regan, and Wong [2]

Received by the editors April 16, 2001 and, in revised form, October 2, 2001.

2000 Mathematics Subject Classification. Primary 34B15.

Key words and phrases. Boundary value problems, existence of positive solutions, higher order equations, multiple solutions, nonlinear equations. 
The following two theorems giving sufficient conditions for the existence of three symmetric positive solutions of the BVPs (1)-(2) and (3)-(4) were obtained in [10].

Theorem A. Let $0<a<b<c / 2$, and suppose $f$ satisfies

$$
\begin{aligned}
& f(w)<8 a \text { for } 0 \leq w \leq a, \\
& f(w) \geq 16 b \text { for } b \leq w \leq 2 b, \\
& f(w) \leq 8 c \text { for } 0 \leq w \leq c .
\end{aligned}
$$

Then the boundary value problem (3)-(4) has three symmetric positive solutions.

Theorem B. Let $0<e<1 / 2$ and define

$$
k_{m}=\left(\int_{0}^{1} G_{m}(1 / 2, s) d s\right)^{-1}, l_{m e}=\left(\int_{e}^{1-e} G_{m}(e, s) d s\right)^{-1},
$$

and

$$
C_{m e}=\max _{0 \leq t \leq 1} \frac{G_{m}(1 / 2, t)}{G_{m}(e, t)}
$$

Let $0<a<b<C_{m e}^{-1} c$ and suppose $f$ satisfies

(B1) $0 \leq f(w)<k_{m} a$ for $0 \leq w \leq a$,

(B2) $f(w) \geq l_{m e} b$ for $b \leq w \leq C_{m e} b$,

(B3) $\quad 0 \leq f(w)<k_{m} c$ for $0 \leq w \leq c$.

Then the boundary value problem (1)-(2) has at least three symmetric positive solutions.

In a recent paper, Baxley and Haywood [5] consider the second order problem (3)-(4) and extend Henderson and Thompson's result to any odd number of symmetric nonnegative solutions. As they point out, "positive" in the conclusions of Theorems A and B above really means nonnegative. They note that if the nonlinear function $f$ is identically zero for $0 \leq x \leq a$ (see Theorem A above), then the smaller solution must in fact be the trivial one. While the analysis used by Baxley and Haywood [5] to obtain their results is quite nice, it does not extend to higher order equations.

Motivated by these results, in this paper we establish some new sufficient conditions that guarantee the existence of any number of symmetric (strictly) positive solutions of the higher order BVP (1)-(2). Section 2 contains some definitions and lemmas that are needed in the remainder of the paper. In Section 3 we prove our main results. The following concepts will also be utilized.

Definition. If $x:[0,1] \rightarrow R$ is a continuous function and $x(t)=x(1-t)$ for $t \in[0,1]$, then we say $x$ is symmetric on $[0,1]$.

Recall that the Green's function for the problem

$$
y^{\prime \prime}=0, y(0)=y(1)=0
$$

is given by

$$
\begin{gathered}
G_{1}:[0,1] \times[0,1] \rightarrow[0, \infty), \\
G_{1}(t, s)= \begin{cases}t(1-s), & t \leq s, \\
s(1-t), & s \leq t .\end{cases}
\end{gathered}
$$


If for $n \geq 2$, we define

$$
G_{n}(t, s)=\int_{0}^{1} G_{1}(t, v) G_{n-1}(v, s) d v,
$$

then $G_{m}(t, s)$ is the Green's function for problem (1)-(2). The equivalent integral equation for $(1)-(2)$ is

$$
x(t)=\int_{0}^{1} G_{m}(t, s) f(x(s)) d s, \quad t \in[0,1] .
$$

\section{Notation AND LEMmas}

Define

$$
J:[0,1] \times[0,1] \rightarrow[0, \infty)
$$

by

$$
J(t, s)= \begin{cases}t, & t \leq s \\ s, & s \leq t\end{cases}
$$

Also define

$$
\begin{aligned}
a_{1}(t) & =t \\
b_{1}(t) & =1 \\
a_{n+1}(t) & =\frac{\int_{0}^{1} J(t, s) a_{n}(s) d s}{\int_{0}^{1} J(1, s) a_{n}(s) d s}, n \geq 1, \\
b_{n+1}(t) & =\frac{\int_{0}^{1} J(t, s) b_{n}(s) d s}{\int_{0}^{1} J(1, s) b_{n}(s) d s}, n \geq 1 .
\end{aligned}
$$

It is easy to verify that $a_{n}(t)$ has the following properties:

$$
\begin{aligned}
a_{n}^{(2 i)}(0)=a_{n}^{(2 i+1)}(1) & =0, \text { for } i=0,1, \cdots, n-2, \\
a_{n}(1) & =1, \\
a_{n}^{(2 n-2)}(0) & =0 \\
(-1)^{n+1} a_{n}^{(2 n-1)}(1) & >0 \\
a_{n}^{(2 n)} & =0 \text { on }(0,1) .
\end{aligned}
$$

In addition, $b_{n}(t)$ satisfies:

$$
\begin{aligned}
b_{n}^{(2 i)}(0)=b_{n}^{(2 i+1)}(1) & =0, \text { for } i=0,1, \cdots, n-2, \\
b_{n}(1) & =1 \\
(-1)^{n+1} b_{n}^{(2 n-2)}(0) & >0 \\
b_{n}^{(2 n-1)}(1) & =0 \\
b_{n}^{(2 n)} & =0 \text { on }(0,1) .
\end{aligned}
$$

The functions $a_{n}(t)$ and $b_{n}(t)$ will be used to estimate the solutions of the boundary value problem (1)-(2). 
Lemma 2.1. Let $z \in C^{2}[0,1]$.

(i) If $z^{\prime \prime}<0$ on $(0, r)$ and $z^{\prime \prime}>0$ on $(r, 1)$ for some $r \in(0,1)$, and $z(0)=z(1)=$ $z^{\prime}(1)=0$, then $z(t)>0$ on $(0,1)$.

(ii) If $z^{\prime \prime}<0$ on $(0, r), z^{\prime \prime}>0$ on $(r, 1)$, and $z(0)=z(s)=z^{\prime}(1)=0$ for some $r$, $s \in(0,1)$, then $z(t)>0$ on $(0, s)$ and $z(t)<0$ on $(s, 1)$.

(iii) If $z^{\prime \prime}<0$ on $(0,1), z(0)=z(r)=0$ for some $r \in(0,1)$, and $z(1)<0$, then $z(t)>0$ on $(0, r)$ and $z(t)<0$ on $(r, 1)$.

(iv) If $z^{\prime \prime}>0$ on $(0,1), z(0)>0$, and $z(r)=z(1)=0$ for some $r \in(0,1)$, then $z(t)>0$ on $(0, r)$ and $z(t)<0$ on $(r, 1)$.

The proof of Lemma 2.1 is straightforward and is left to the reader. Now we can use Lemma 2.1 to prove the following lemma.

Lemma 2.2. Suppose $x \in C^{2 n}[0,1]$ is nonnegative on $[0,1],(-1)^{n} x^{(2 n)}(t)$ is nonnegative on $[0,1]$, and

$$
x^{(2 i)}(0)=x^{(2 i+1)}(1)=0, \text { for } i=0,1, \cdots, n-1 .
$$

Then

$$
a_{n}(t) x(1) \leq x(t) \leq b_{n}(t) x(1) \text { on }[0,1]
$$

Proof. Define

$$
d_{n}(t)=x(t)-a_{n}(t) x(1)
$$

It is easy to verify that

$$
\begin{aligned}
d_{n}^{(2 i)}(0) & =0, \quad i=0,1,2, \cdots, n-2, \\
d_{n}^{(2 i+1)}(1) & =0, \quad i=0,1,2, \cdots, n-2, \\
d_{n}(1) & =0, \\
(-1)^{n+1} d_{n}^{(2 n-2)}(0) & =0 \\
(-1)^{n+1} d_{n}^{(2 n-1)}(1) & <0, \\
(-1)^{n+1} d_{n}^{(2 n)}(0) & <0 \text { on }(0,1) .
\end{aligned}
$$

By Rolle's theorem and induction, we see that there exist $r_{1}, r_{2}, \cdots, r_{2 n-2} \in(0,1)$ such that

$$
d_{n}^{(i)}\left(r_{i}\right)=0, \text { for } i=1,2, \cdots, 2 n-2 .
$$

Applying Lemma 2.1(iii) to $(-1)^{n+1} d_{n}^{(2 n-2)}$, we have

$$
(-1)^{n+1} d_{n}^{(2 n-2)}>0 \text { on }\left(0, r_{2 n-2}\right) \text { and }(-1)^{n+1} d_{n}^{(2 n-2)}<0 \text { on }\left(r_{2 n-2}, 1\right) .
$$

Next, we apply Lemma 2.1(ii) to $(-1)^{n+2} d_{n}^{(2 n-4)}$ to obtain

$$
(-1)^{n+2} d_{n}^{(2 n-4)}>0 \text { on }\left(0, r_{2 n-4}\right) \text { and }(-1)^{n+2} d_{n}^{(2 n-4)}<0 \text { on }\left(r_{2 n-4}, 1\right) .
$$

Continuing this procedure, we finally arrive at

$$
(-1)^{2 n-1} d_{n}^{\prime \prime}>0 \text { on }\left(0, r_{2}\right) \text { and }(-1)^{2 n-1} d_{n}^{\prime \prime}<0 \text { on }\left(r_{2}, 1\right),
$$

i.e.,

$$
d_{n}^{\prime \prime}>0 \text { on }\left(0, r_{2}\right) \text { and } d_{n}^{\prime \prime}<0 \text { on }\left(r_{2}, 1\right) .
$$

Applying Lemma 2.1(i) to $d_{n}$, we have

$$
d_{n}>0 \text { on }(0,1)
$$


which implies that

$$
x(t) \geq a_{n}(t) x(1) \text { on }[0,1] .
$$

Similarly, we can show that

$$
x(t) \leq b_{n}(t) x(1) \text { on }[0,1]
$$

to complete the proof of the lemma.

Next, we define

$$
A_{n}(t)= \begin{cases}a_{n}(2 t), & 0 \leq t \leq \frac{1}{2} \\ a_{n}(2-2 t), & \frac{1}{2} \leq t \leq 1\end{cases}
$$

and

$$
B_{n}(t)= \begin{cases}b_{n}(2 t), & 0 \leq t \leq \frac{1}{2}, \\ b_{n}(2-2 t), & \frac{1}{2} \leq t \leq 1 .\end{cases}
$$

Note that $A_{n}(t)$ and $B_{n}(t)$ are symmetric on $[0,1] ; A_{n}(t)$ and $B_{n}(t)$ will be used to estimate the solutions of the problem (1)-(2).

Lemma 2.3. If $x \in C^{2 m}[0,1]$ and $x(t)$ is symmetric on $[0,1]$, then

$$
x^{(2 i+1)}(1 / 2)=0, \quad i=0,1,2, \cdots, m-1 .
$$

Proof. Assume to the contrary that the conclusion of the lemma is not true; then $\left\{0 \leq i \leq m-1: x^{(2 i+1)}(1 / 2) \neq 0\right\}$ is not empty, so we let $k=\min \{0 \leq i \leq m-1$ : $\left.x^{(2 i+1)}(1 / 2) \neq 0\right\}$. Then, by Taylor's formula, we have

$$
x\left(\frac{1}{2}+t\right)=\sum_{i=0}^{k} \frac{1}{(2 i) !} x^{(2 i)}\left(\frac{1}{2}\right) t^{2 i}+\frac{1}{(2 k+1) !} x^{(2 k+1)}\left(\frac{1}{2}\right) t^{2 k+1}+o\left(t^{2 k+1}\right)
$$

and

$$
x\left(\frac{1}{2}-t\right)=\sum_{i=0}^{k} \frac{1}{(2 i) !} x^{(2 i)}\left(\frac{1}{2}\right) t^{2 i}-\frac{1}{(2 k+1) !} x^{(2 k+1)}\left(\frac{1}{2}\right) t^{2 k+1}+o\left(t^{2 k+1}\right) .
$$

Hence,

$$
x\left(\frac{1}{2}+t\right)-x\left(\frac{1}{2}-t\right)=\frac{2}{(2 k+1) !} x^{(2 k+1)}\left(\frac{1}{2}\right) t^{2 k+1}+o\left(t^{2 k+1}\right) .
$$

So if $t>0$ is small enough, then

$$
x\left(\frac{1}{2}+t\right) \neq x\left(\frac{1}{2}-t\right) .
$$

This contradicts the fact that $x$ is symmetric on $[0,1]$ and completes the proof of the lemma.

Lemma 2.4. Suppose $x \in C^{2 m}[0,1]$ is symmetric on $[0,1], x^{(2 i)}(0)=x^{(2 i)}(1)=$ $0, i=0,1,2, \cdots, m-1,(-1)^{m} x^{(2 m)} \geq 0$ on $[0,1]$, and $x(t) \geq 0$ on $[0,1]$. Then,

$$
x(1 / 2) A_{m}(t) \leq x(t) \leq x(1 / 2) B_{m}(t) \text { for } 0 \leq t \leq 1 .
$$


Proof. Define

$$
y(t)=x(t / 2)
$$

then Lemma 2.3 implies

$$
y^{(2 i+1)}(1)=0, \quad i=0,1, \cdots, m-1 .
$$

Also, we have

$$
(-1)^{m} y^{(2 m)}(t) \geq 0 \quad \text { on } \quad[0,1]
$$

and

$$
y^{(2 i)}(0)=0, \quad i=0,1,2, \cdots, m-1 .
$$

Applying Lemma 2.2, we obtain

$$
a_{m}(t) y(1) \leq y(t) \leq b_{m}(t) y(1) \text { on }[0,1],
$$

which implies that

$$
A_{m}(t) x(1 / 2) \leq x(t) \leq B_{m}(t) x(1 / 2) \text { on }[0,1 / 2] .
$$

Similarly, we can prove that

$$
A_{m}(t) x(1 / 2) \leq x(t) \leq B_{m}(t) x(1 / 2) \text { on }[1 / 2,1],
$$

and this completes the proof of the lemma.

Corollary 2.5. If $x(t)$ is a symmetric positive solution of problem $(1)-(2)$, then

$$
x(1 / 2) A_{m}(t) \leq x(t) \leq x(1 / 2) B_{m}(t), \text { for } 0 \leq t \leq 1 .
$$

Corollary 2.5 follows immediately from Lemma 2.4. Lemma 2.4 and Corollary 2.5 will be used to estimate and control the solutions of problem (1)-(2). In addition, they are interesting results in their own right.

\section{MAIN RESULTS}

The following fixed point theorem will be used to prove our main results.

Theorem C (Krasnosel'skii's Fixed Point Theorem [11]). Let $\mathcal{B}$ be a Banach space and let $\mathcal{P} \subset \mathcal{B}$ be a cone in $\mathcal{B}$. Assume that $\Omega_{1}$ and $\Omega_{2}$ are open subsets of $\mathcal{B}$ with $0 \in \Omega_{1} \subset \overline{\Omega_{1}} \subset \Omega_{2}$, and let

$$
L: \mathcal{P} \cap\left(\overline{\Omega_{2}}-\Omega_{1}\right) \rightarrow \mathcal{P}
$$

be a completely continuous operator such that either

(C1) $\quad\|L u\| \leq\|u\|$ if $u \in \mathcal{P} \cap \partial \Omega_{1}$, and $\|L u\| \geq\|u\|$ if $u \in \mathcal{P} \cap \partial \Omega_{2}$, or

(C2) $\quad\|L u\| \geq\|u\|$ if $u \in \mathcal{P} \cap \partial \Omega_{1}$, and $\|L u\| \leq\|u\|$ if $u \in \mathcal{P} \cap \partial \Omega_{2}$.

Then $L$ has a fixed point in $\mathcal{P} \cap\left(\overline{\Omega_{2}}-\Omega_{1}\right)$.

Next, we must define the appropriate spaces. Let $\mathcal{B}=C[0,1]$ be endowed with norm

$$
\|x\|=\max _{t \in[0,1]}|x(t)|, x \in \mathcal{B} .
$$

Then $\mathcal{B}$ is a Banach space, and motivated by the estimates in Corollary 2.5, we take

$$
\mathcal{P}=\left\{\begin{array}{l|l}
x \in \mathcal{B} & \begin{array}{l}
x(t) \text { is nonnegative and symmetric on }[0,1] \\
A_{m}(t) x(1 / 2) \leq x(t) \leq B_{m}(t) x(1 / 2) \text { on }[0,1]
\end{array}
\end{array}\right\}
$$


to be our positive cone in $\mathcal{B}$. Define the operator $T: \mathcal{B} \rightarrow \mathcal{B}$ by

$$
(T x)(t)=\int_{0}^{1} G_{m}(t, s) f(x(s)) d s .
$$

Then, solving the boundary value problem (1)-(2) is equivalent to finding a solution of

$$
x=T x, x \in \mathcal{B} .
$$

From Lemma 2.4, we see that $T(\mathcal{P}) \subset \mathcal{P}$, and it is easy to check that $T: \mathcal{P} \rightarrow \mathcal{P}$ is a completely continuous operator. Thus, to solve problem (1)-(2), it suffices to find a fixed point of $T$ in $\mathcal{P}$.

Next, we define some useful constants. For each $m \geq 1$, let

$$
K_{m}=\int_{0}^{1} G_{m}(1 / 2, s) d s
$$

and for each $m \geq 1$ and $r \in(0,1 / 2)$, let

$$
L_{m}(r)=\int_{r}^{1-r} G_{m}(1 / 2, s) d s .
$$

The following two lemmas will be used in the proof of our main results.

Lemma 3.1. If $a>0, f(z)<a / K_{m}$ for $z \in[0, a]$, and $x \in \mathcal{P}$ with $\|x\|=a$, then $\|T x\|<a$.

Proof. If $x \in \mathcal{P}$ with $\|x\|=a$, then

$$
\|T x\|=(T x)(1 / 2) \leq \int_{0}^{1} G_{m}(1 / 2, s) f(x(s)) d s<\left(a / K_{m}\right) \int_{0}^{1} G_{m}(1 / 2, s) d s=a
$$

which proves the lemma.

Lemma 3.2. If $b>0, r \in(0,1 / 2), f(z)>\frac{b}{L_{m}(r)}$ for $z \in\left[b A_{m}(r), b\right], x \in \mathcal{P}$, and $\|x\|=b$, then $\|T x\|>b$.

Proof. If $t \in[r, 1-r]$, then

$$
T x(t) \geq A_{m}(t)\|x\| \geq A_{m}(r)\|x\| .
$$

Thus, we have

$$
\begin{aligned}
\|T x\|=T x(1 / 2) & =\int_{0}^{1} G_{m}(1 / 2, s) f(x(s)) d s \geq \int_{r}^{1-r} G_{m}(1 / 2, s) f(x(s)) d s \\
& >\frac{b}{L_{m}(r)} \int_{r}^{1-r} G_{m}(1 / 2, s) d s=b .
\end{aligned}
$$

This completes the proof of the lemma.

Now we are ready to prove our main results. In what follows we let $k$ be a positive integer.

Theorem 3.3. If there are constants $0<a_{1}<a_{2}<\cdots<a_{2 k}$ and $r_{1}, r_{3}, \ldots$, $r_{2 k-1} \in(0,1 / 2)$ such that

(1) $f(z)<a_{i} / K_{m}$ for $z \in\left[0, a_{i}\right], i=2,4, \ldots, 2 k$, and

(2) $f(z)>\frac{a_{i}}{L_{m}\left(r_{i}\right)}$ for $z \in\left[a_{i} A_{m}\left(r_{i}\right), a_{i}\right], i=1,3, \ldots, 2 k-1$,

then the boundary value problem (1)-(2) has at least $2 k-1$ symmetric positive solutions. 
Proof. Define

$$
\Omega_{i}=\left\{x \in \mathcal{B}:\|x\|<a_{i}\right\}, \quad i=1,2, \ldots, 2 k .
$$

Then, from Lemmas 3.1 and 3.2, we have

$$
\|T u\|<\|u\| \text { for } u \in \mathcal{P} \cap \partial \Omega_{i}, i=2,4, \ldots, 2 k,
$$

and

$$
\|T u\|>\|u\| \text { for } u \in \mathcal{P} \cap \partial \Omega_{i}, i=1,3, \ldots, 2 k-1 .
$$

Now from Theorem $\mathrm{C}$ we see that $T$ has $2 k-1$ fixed points, one in each of the sets $\mathcal{P} \cap\left(\overline{\Omega_{j}}-\Omega_{j-1}\right), j=2,3, \ldots, 2 k$. This completes the proof of the theorem.

The corresponding result for an even number of solutions is as follows.

Theorem 3.4. If there are constants $0<a_{1}<a_{2}<\cdots<a_{2 k+1}$ and $r_{1}, r_{3}, \ldots$, $r_{2 k-1} \in(0,1 / 2)$ such that

(1) $f(z)<a_{i} / K_{m}$ for $z \in\left[0, a_{i}\right], i=2,4, \ldots, 2 k$, and

(2) $f(z)>\frac{a_{i}}{L_{m}\left(r_{i}\right)}$ for $z \in\left[a_{i} A_{m}\left(r_{i}\right), a_{i}\right], i=1,3, \ldots, 2 k+1$,

then the boundary value problem (1)-(2) has at least $2 k$ symmetric positive solutions.

Proof. Define

$$
\Omega_{i}=\left\{x \in \mathcal{B}:\|x\|<a_{i}\right\}, \quad i=1,2, \ldots, 2 k+1 .
$$

Then, from Lemmas 3.1 and 3.2, we have

$$
\|T u\|<\|u\| \text { for } u \in \mathcal{P} \cap \partial \Omega_{i}, i=2,4, \ldots, 2 k,
$$

and

$$
\|T u\|>\|u\| \text { for } u \in \mathcal{P} \cap \partial \Omega_{i}, i=1,3, \ldots, 2 k+1 .
$$

Now from Theorem $\mathrm{C}$ we see that $T$ has $2 k$ fixed points, one in each of the sets $\mathcal{P} \cap\left(\overline{\Omega_{j}}-\Omega_{j-1}\right), j=2,3, \ldots, 2 k+1$. This completes the proof of the theorem.

In a similar fashion we can prove the following result.

Theorem 3.5. If there are constants $0<a_{1}<a_{2}<\cdots<a_{2 k}$ and $r_{2}, r_{4}, \ldots$, $r_{2 k} \in(0,1 / 2)$ such that

(1) $f(z)<a_{i} / K_{m}$ for $z \in\left[0, a_{i}\right], i=1,3, \ldots, 2 k-1$, and

(2) $f(z)>\frac{a_{i}}{L_{m}\left(r_{i}\right)}$ for $z \in\left[a_{i} A_{m}\left(r_{i}\right), a_{i}\right], i=2,4, \ldots, 2 k$,

then the boundary value problem (1)-(2) has at least $2 k-1$ symmetric positive solutions.

We leave the formulation of the analogous result corresponding to Theorem 3.4 to the reader.

In conclusion, we would like to return to some of the ideas discussed in the Introduction. In [10], Henderson and Thompson used three inequalities to guarantee the existence of three symmetric positive solutions, one of which may in fact be the trivial solution. Baxley and Haywood [5] added a fourth condition and as a consequence were able to guarantee that there were at least three strictly positive solutions. The approach used here allows us to obtain the existence of $n$ symmetric (strictly) positive solutions by imposing $n+1$ conditions.

As to the various constants involved, in Theorems 3.3-3.5, the roles played by the $r_{j}$ 's are the same as that of the $e$ in Theorem B above; however, we allow, for 
example, that $r_{1}=r_{3}$ in Theorem 3.3, so they are more flexible to use. The constant $k_{m}$ in Theorem B is the same as our $K_{m}$. The constant $1 / l_{m e}$ in Theorem B is similar to our $L_{m}\left(r_{i}\right)$, but when $e=r, 1 / l_{m e}<L_{m}(r)$, so $l_{m e}>1 / L_{m}(r)$. Thus, our condition $f(z)>b / L_{m}(r)$ for $z \in\left[b A_{m}(r), b\right]$ is better than (B2) in Theorem B. While our $A_{m}(r)$ is similar to $1 / C_{m e}$ in Theorem $\mathrm{B}$, a very good estimate was used to obtain $A_{m}(r)$, and as a consequence, we believe that our condition is better.

\section{ACKNOWLEDGMENT}

The authors would like to thank the referee for making several helpful suggestions that significantly improved this paper.

\section{REFERENCES}

[1] R. P. Agarwal, Focal Boundary Value Problems for Differential and Difference Equations, Kluwer Academic, Dordrecht, 1998. MR 99h:34036

[2] R. P. Agarwal, D. O’Regan, and P. J. Y. Wong, Positive Solutions of Differential, Difference, and Integral Equations, Kluwer Academic, Dordrecht, 1999. MR 2000a:34046

[3] R. Agarwal and F. H. Wong, Existence of positive solutions for higher order boundary value problems, Nonlinear Studies 5 (1998), 15-24. MR 99e:34024

[4] R. I. Avery, J. M. Davis, and J. Henderson, Three symmetric positive solutions for Lidstone problems by a generalization of the Leggett-Williams theorem, Electron. J. Differential Equations, Vol. 2000 (2000), No. 40, pp 1-15. MR 2001c:34048

[5] J. Baxley and L. J. Haywood, Nonlinear boundary value problems with multiple solutions, Nonlinear Anal. 47 (2001), 1187-1198.

[6] J. Baxley and L. J. Haywood, Multiple positive solutions of nonlinear boundary value problems, Dynam. Contin. Discrete Impuls. Systems, to appear.

[7] P. W. Eloe, Nonlinear eigenvalue problems for higher order Lidstone boundary value problems, Electron. J. Differential Equations 2000 No. 2, 1-8. MR 2001a:34032

[8] P. W. Eloe and J. Henderson, Positive solutions for higher order ordinary differential equations, Electron. J. Differential Equations 1995 (1995), 1-8. MR 96a:34037

[9] L. H. Erbe, S. Hu, and H. Y. Wang, Multiple positive solutions of some boundary value problems, J. Math. Anal. Appl. 184 (1994), 640-648. MR 95f:34023

[10] J. Henderson and H. B. Thompson, Multiple symmetric positive solutions for a second order boundary value problem, Proc. Amer. Math. Soc. 128 (2000), 2373-2379. MR 2000k:34042

[11] M. A. Krasnosel'skii, Positive Solutions of Operator Equations, Noordhoff, Groningen, 1964. MR 31:6107

[12] R. Ma, J. Zhang, and S. Fu, The method of lower and upper solutions for fourth-order twopoint boundary value problems, J. Math. Anal. Appl. 215 (1997), 415-422. MR 98i:34037

[13] P. J. Y. Wong and R. Agarwal, Eigenvalues of Lidstone boundary value problems, Appl. Math. Comput. 104 (1999), 15-31. MR 2000b:34034

Department of Mathematics, University of Tennessee at Chattanooga, Chattanooga, Tennessee 37403

E-mail address: john-graef@utc.edu

Department of Mathematics and Statistics, Mississippi State University, Mississippi State, Mississippi 39762

E-mail address: qian@math.msstate.edu

Department of Mathematics and Statistics, Mississippi State University, Mississippi State, Mississippi 39762

E-mail address: by2@ra.msstate.edu 